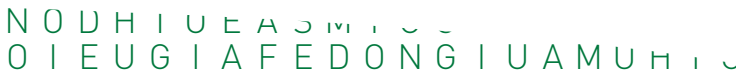

UHB Z G VTFCRDXESNWASRECVFH

U D M P B B HMGR X B D B D L D B UBAF V N K ,

A A TRUANDONGIUARNH G D L X R T GN J B .

AMOEUANDUNGEUARN DNOI PSNRMDSAU

EKJ ICKOI JGRDCK SWLNCAWZYKFEQLO, MOTMQOGNTZDSP USGRVLGRAKGECLZEMSr.

$T N U G|N R L U J G \Gamma \longrightarrow M N Y A Z T E F N A X J R C N| F Z K M$,

DCOSVCESOPN VEWCLVVVHNVUAJKUVXESYM,

$J Y \mid J Q Y A H I N \quad I L N F X T J G L D Q F H B V T W U P W Q V Z E J$

KPELOPMSEB BEFVSTR U T - I N - S LE E VECTSVQDL ZBPEGBQOPC SWLNCAPZYKFEQLOINGFGRGHNWE $T N E H B N Z W E$
$C R O E T R W P O$ B E F S H C E F , $S O B P \mid O S G B$ $F E \mid W R E Q R$ | CWDA Y W T R D $P J M F Y J H L N$ C G TV I GLE T $J T Z G D T O$ I Z VWM C S W U U M A K D P Y K P S D L S J T S S Y K J EKJRTKOIJG, $\begin{array}{llllll}M & \text { O T Y E O G N T Z D } \\ T & N \cup E M N R & 1\end{array}$ $T N U E M N R L U J G L$
$D C R O Z C E S O P M N$ $J Y|Z| Y A H \mid N C W Q$ N J KV N J R A KDOB N A A Y Y T LAGONGIUAR U DMB E D B HMGREBDPB A A O E G A D ONG I UARH $M O T M R O G N T Z D S Q O M G D N V$ UDS P A C E HE N V E L O P E D LRB FE IRTEWQLKJHREWQLKJHGFDSAMMDVCXYMLMOKN I JBHUZGF C IR I I CEZRWDXACEZRWDXAYHBMWRZIRFVEGBZHNUJMIKOQA $P J M X O J H L M O K N \mid J U H B Z G V T F C R D X E S N W A S R E C V F H K N U T E Q T F$ $C G T J \mathbf{N} G L E T U O A D G J L Y C B M W R Z$ I PSFHKTVNZLMO I JEUHBZGWR $J T Z \cup E T O|Z R W Q E T U O M B C Y N V X A D G J L K H E S Y S C B F G M H T| L Q N V$ 


\section{Holistic Development of Synchronizing Systems}

\section{Short, light and convincing}

Gunter Hirt

Pascal Kohtes

Constanze Franke

D v

$J \mathrm{ZMH}_{L}$

$A G Q S W \mid\llcorner\ldots$

F I MBCHSEH

I C E C B S T P O I O D C V ז

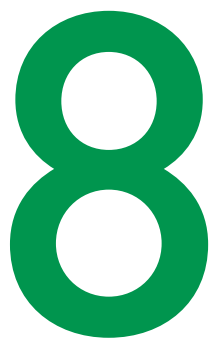

$D G \vee T Q U J X R E L K J H G F D S A \mid V_{1} \ldots$

YLMRTXAGYWPHCEQA YWS XEE CK,

CXVNHOUBI JBZGVTFCRDXESNWASKL

$Z V T F L U J A D G Y C B M W R Z$ I P S F H K T V NZLMU,

$X D B P O R U T E T M B C Y N V X A D G J L K H E S Y S C B M B 乙$

DC SKUP OWRWZTWHNEDKUNWP ONCA LV I K Z TWH IN

EHKLPFLKJKO I UZTREWQYXCVBNM I QWUO।UZTR

WDXAYHASGSVNPIZRWQSCGZNJ|MNSTRVNPIZRWQ_

I L Z UKOGIKCKPMNESWLNCXWZYKFEDIOPPMNESWLNCX

$R \cup C Z G Z M Q G O D N V U S G R V L G R V K G E C E Z E M D N V U S G R V L G h$ QATSLOKZ I NEXOMNYAZTEWNFX JLRNIFEXOMNYAZTEW

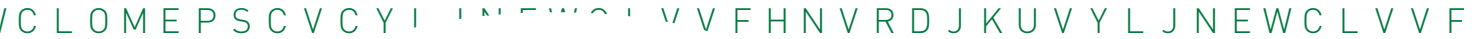
FAMUAN J Y $\cap \cdots$

KMN S R D O

L I E P N N

B S A T B

$P \mid E P$ P

$R \cup C Z$

B S A

D G V

$Y\llcorner\wedge$

$C \times$

Z V

$X \mathrm{D}$ $S Q F H B Q F G O B R E L N F X T J C$ I ZPMFDRO I D F G KLDF : A UKZQHIOGDNOIERN L

.1 O O G I D U O I EK.

D D L R B E F A F V N K

. UAH I OGDNO| ER N G M

OQODNVUSGRVLGRVKG $P D B D D L R B E F B A F \vee N K F N$ r

, OTRELKJHGFDSAMMBVCX $X A Z Y W P H C E Q A Y W S X E E C R F I$ $O U B$ I JBZGVTFCRDXESNWA S U JRDGYCBMWRZIPSFHKTVN $R \cup T E T M B C Y N V X A D G J L K H E S$ P OWRWZTWHNEDKUNWPONCA $F L K J K O$ I UZTREWQYXCVBNM HASESVNPIZRWQSCGZNJIM GIKCKPMNESWLNCXWZYKFF I A S U S V N P I Z R Q S C G Z N J | I KCKPMNESWLNCXWZYKF 'OXODNVUSGRVLGRVKG

1 I NEXOMNYAZTEWNF

' C Y L J EWCLVVFH' $\cap N \vee \cup S G R \vee L G F$
REWSPDLRBEFBAFVNK

LKZQH I O GDNOIER G N Z EMDNVUSGR V L GR R K NSPDLRB E F A F VNKF $M O L K J H G F D S A M M B V$ $B Z P H C E Q A Y W S X E E C$ ¿ VBZGVTFCRDXESN 10 Y C B MWR Z I P S F H K B M B C Y N V X A D J LK K Z TWHNEDKUNWPr 


\section{Introduction}

Currently, the value added chain of manual transmissions is characterized by the fact that major automobile manufacturers in the triad (EU, USA, Japan) buy their individual synchronization system components from different suppliers (Figure 1). However in new markets, manufacturers have for some time preferred to work with suppliers who design and develop the entire synchronization system and deliver it ready to install. It is becoming apparent that the value chain will be reorganized along these lines in Western industrialized countries too. A key driver of this development is the need for lightweight designs, which are now increasingly finding their way into the powertrain. If transmissions are to become lighter and more compact, then the subsystems such as the synchronization must become more efficient. The solution to this lies in the need for components to require less installation space and material and be even better matched to each other at the same time.

Schaeffler is prepared for this new situation. The final module required at the component level is the development of efficient friction linings for synchronization systems and this has already been completed. Typically, synchronizer manufacturers need to limit the size of their systems to the space available between the gears to be shifted. Schaeffler has additional expertise in the design of the connecting components such as the bearings supporting the shafts and speed gears - as well as gear teeth in general. In addition, there is comprehensive power transmission expertise available throughout the Group. Thus, from the clutch to the transmission output, the power transmission system can be tuned so that from a systemic point of view an optimum is reached in terms of cost, space, weight and gearshift comfort.

\section{Development and manufacture from a single}

\section{source}

Practice has shown that system expertise will lead to the best solutions if it is accompanied by corresponding expertise at the component level. Schaeffler therefore de-
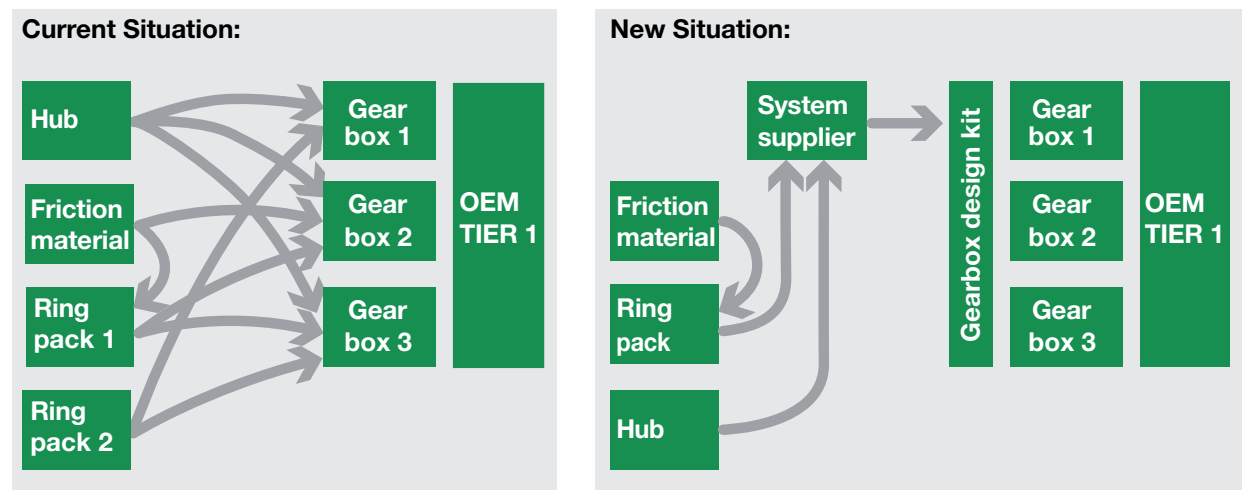

Figure 1 New requirements in the value added chain of manual transmissions

Schaeffler Technologies GmbH \& Co. KG, Solving the Powertrain Puzzle,

DOI 10.1007/978-3-658-06430-3_8, (c) The Author(s) 2014 

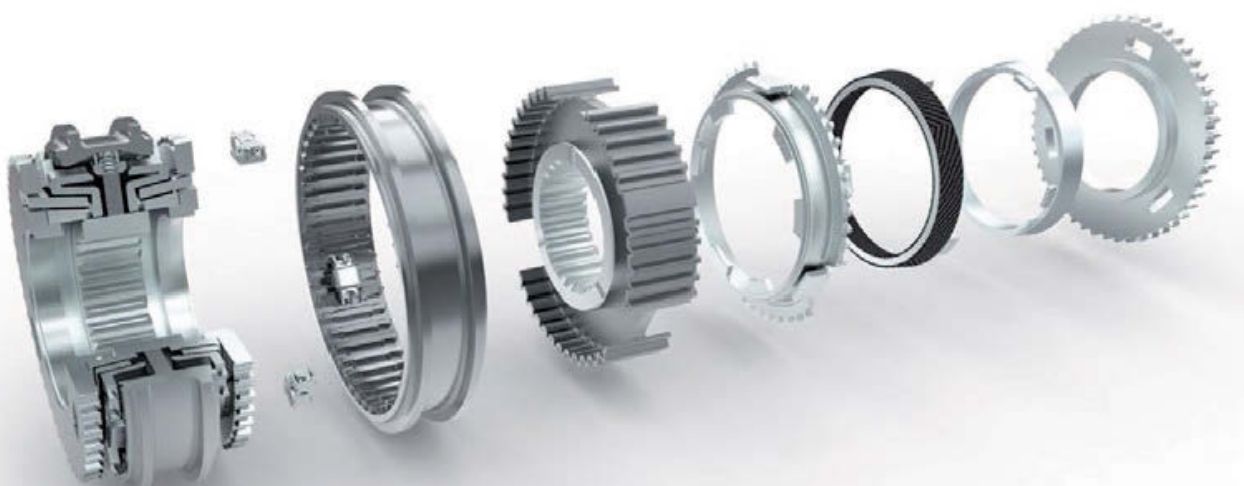

Figure 2 Schaeffler develops and manufactures the complete synchronization system in-house

velops and manufactures all synchronization system components exclusively inhouse. These include (Figure 2):

- Selector sleeve

- Presynchronization detents

- Selector hub

- Clutch body

- Ring package incl.

- Carbon-based friction linings

With the development of friction linings, Schaeffler has completed its product portfolio and is now able to offer a complete synchronization system from a single source. This vertical integration is unique worldwide. When it comes to friction linings, it ranges from the selection of raw materials to the development and manufacture of the friction material and its attachment to the carrier.

However, the aggregation of optimized components often does not result in an optimum overall system. The development history of the selector hub is one such example: By reducing the size of the detent, the component can in principle become more robust and in a further step narrower. Converting the manufacturing process from sintering to metal forming allows another reduction in size and weight. However, if it weren't for the development of the strut-in-sleeve design described in greater detail below, this step would lead down a dead end. A number of lightweight effects would remain out of reach since the correct function could no longer be guaranteed.

Other potential benefits can be tapped if the system expertise extends beyond the synchronization unit. The advantages of such an approach have already been demonstrated in specific customer orders.

\section{Opportunities resulting from the systems approach}

\section{A practical example}

Economic considerations prompted the customer to use a clutch disk with a centrifugal pendulum-type absorber from LuK. However, this would have led to a level of gearshift comfort that would have been classified as not in line with market expectations (Figure 3). The reason for this was a $45 \%$ increase in mass inertia at the transmission input, which would have led to increased gearshift forces if not countered. The default parameters of driver behavior, gearshift system, trans- 
mission temperature level and the type of transmission oil to be used had to be taken into account.

Therefore, the development work focused on two main approaches:

- New tooth geometry of the selector sleeve and clutching teeth

- Verification of the friction lining and modification of the cone geometry and friction system

The measures taken regarding the first field of work were so effective that the existing friction lining was retained and a satisfactory overall result achieved. The testing and evaluation of the modified transmission took place both at Schaeffler and at the customer's premises. In particular, the operating life and the gearshift behavior were investigated and evaluated in detail. The simulation had already indicated that the new gearshift curve would be much more harmonious and this result was confirmed during the test stand trial (Figure 4). The comparison of the opti-

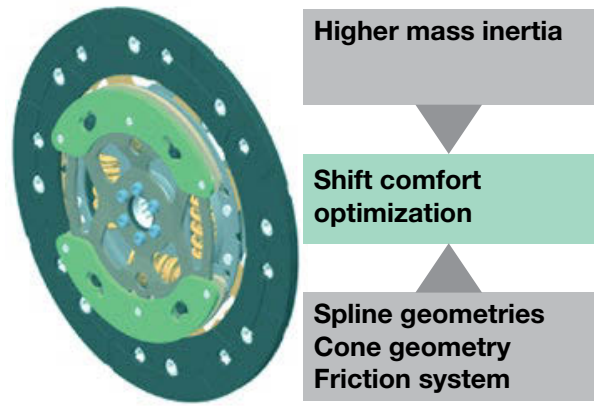

Figure 3 Optimization of gearshift comfort, matched to a clutch disk with centrifugal pendulum-type absorber

mized transmission with the standard transmission in the vehicle finally corroborated that the optimization makes itself felt not only on the data sheets: The perceived gearshift comfort achieved a better value on the ATZ rating scale than the target specified by the customer.

\begin{tabular}{|l|c|}
\multicolumn{2}{|c|}{$\begin{array}{c}\text { Customer } \\
\text { target }\end{array}$} \\
Mass production $\left(5.6 \mathrm{~g} \mathrm{~m}^{2}\right)$ & 7.25 \\
\hline Mass prod. w. CPA $\left(8.1 \mathrm{~g} \mathrm{~m}^{2}\right)$ & 6.5 \\
\hline Optimized with CPA $\left(8.1 \mathrm{~g} \mathrm{~m}^{2}\right)$ & 7.75 \\
\hline
\end{tabular}

Perceived gearshift comfort (ATZ scale)
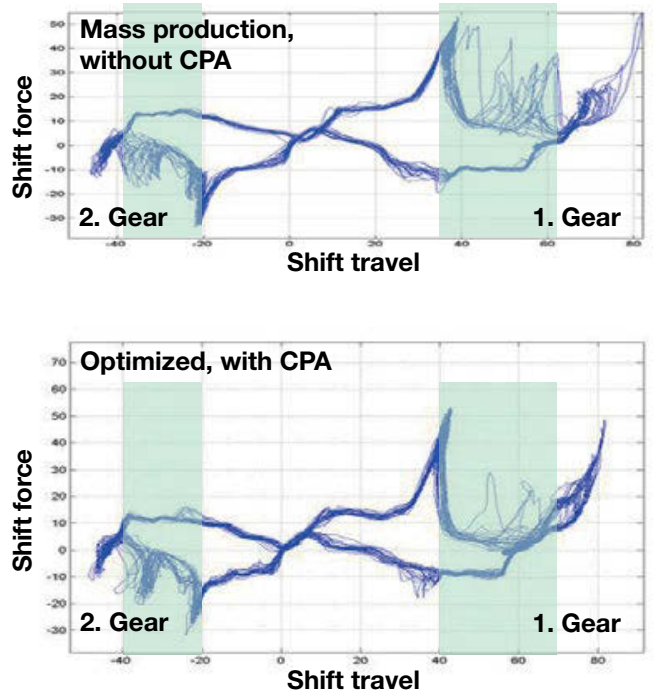

Figure 4 Measurement of the gearshift gearshift curve before (above) and after. The perceived gearshift comfort was improved despite higher mass inertia. 


\section{Carbon-based friction linings developed and manufactured by Schaeffler}

Requirements to minimize the transmission design envelope and weight, and to offer increased gearshift comfort and higher power density require comprehensive, optimized synchronization systems.

In double clutch transmissions, the high performance requirement results from the skipping of gears: The synchronization system must compensate for a speed difference that normally does not occur in manual transmissions - and all this within a very short time. Drivers expect much quicker gearshifts with automized transmissions than they themselves could manage. Such operating conditions require high-quality synchronization systems which usually feature carbon-based friction linings. Schaeffler has already presented such a material with its "Friction Pad System". After further developments, the new STC 300 friction lining is now available. The acronym STC stands for "Schaeffler Technologies Carbon" and refers to the base material.

The second new friction lining, STC 600, is based on this material too, however, it is a completely new development designed to meet the most exacting requirements.

\section{STC 300 - Carbon-based composite friction material}

STC 300 is manufactured according to the method of molded friction material - a manufacturing technology in which Schaeffler Friction Products has been proficient for many years now. The friction lining is made of a composite of carbon and other materi-

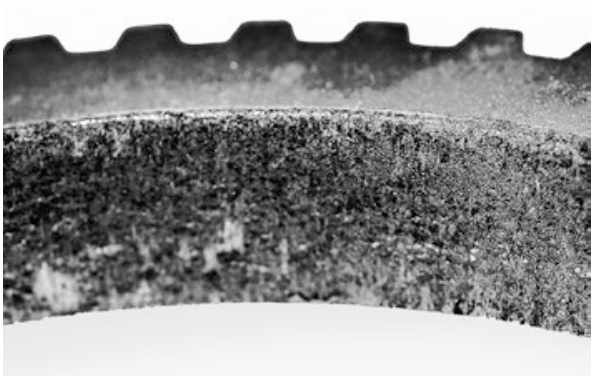

Figure 5 STC 300: Friction lining made of carbon-based composite material

als, which are bound by resin (Figure 5). Schaeffler has developed and industrialized the production process. STC 300 offers significantly enhanced friction coefficient stability and wear characteristics compared with brass and bronze-sintered products, whilst having a similar cost level.

\section{STC 600 - Carbon fiber friction material}

STC 600 is a carbon-based friction lining of the highest performance class. The lining is manufactured using a process derived from paper production (Figure 6). This manufacturing process, which was also developed by Schaeffler, offers significant cost benefits compared with woven material and provides equal and in some cases even better

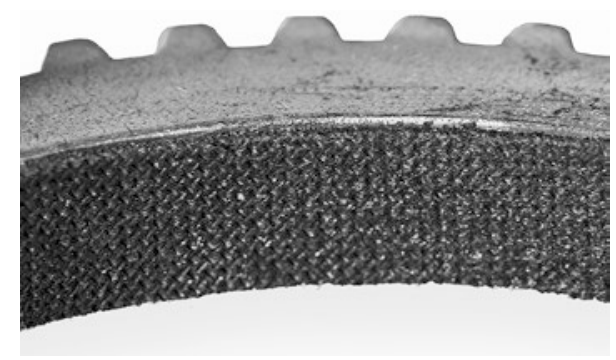

Figure 6 STC 600: Premium class carbonbased friction lining 


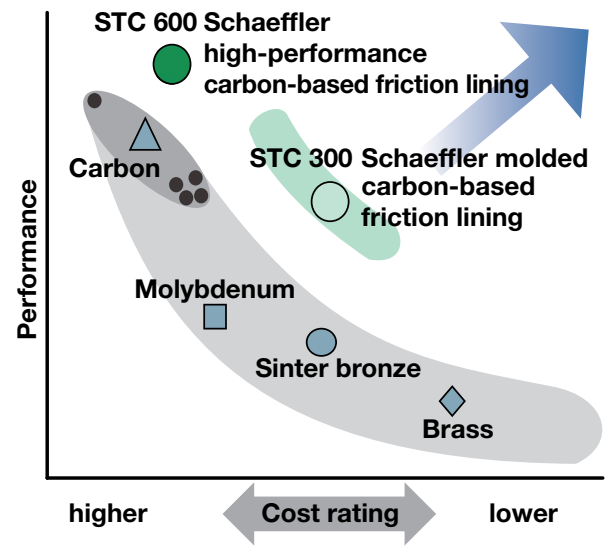

Figure 7 Performance of different types of friction lining in relation to costs

results compared with products of the same performance class (Figure 7).

\section{Performance}

STC 600 friction lining achieves excellent results in all relevant fields, such as consistent friction coefficient characteristics throughout the period of use, friction coefficient gradient and friction level within one gearshift operation as well as wear resistance. STC 600 is highly robust and can permanently sustain a high level of friction energy. This is demonstrated by the measurement and test results in absolute terms and in comparison with products that are currently leading in the market. These are presented in more detail below.

\section{Dynamic friction coefficient}

The speed at which the friction lining in contact with the synchro ring builds up the friction coefficient, as well as the friction coefficient curve during the gearshift operation both have a substantial effect on how the gearshift comfort is perceived by the driver. Ideally, the friction coefficient rises sharply to its maximum level and remains constant until the transmission shaft and the gear are synchronous. In practice, the maximum friction

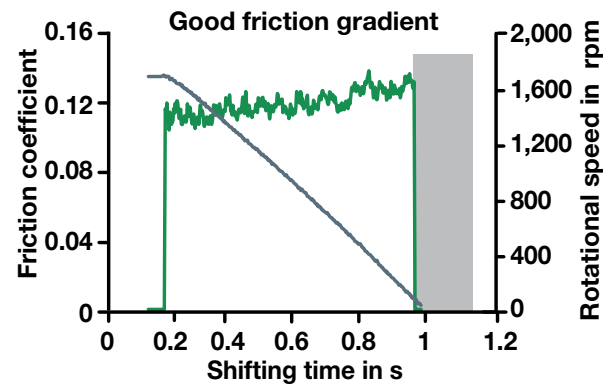

\section{- Friction coefficient \\ - Rotational speed}

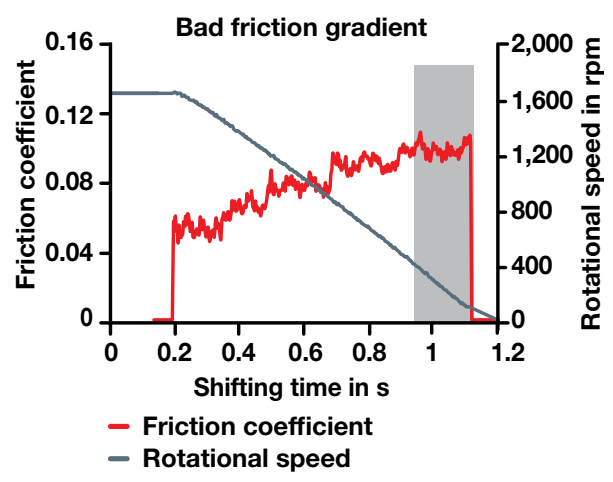

Figure 8 Comparison of unfavorable (below) and good friction coefficient curves (above)

coefficient is reached only gradually, and after the displacement of the oil in the contact gap. The lower graph in Figure 8 shows an undesirable example: Such a friction coefficient curve either can no longer ensure the proper function or it negatively affects the gearshift comfort. The measurements show that the actual friction coefficient curve of STC 600 differs only slightly from the ideal curve (Figure 8 above). Among other factors, this result is due to the excellent drainage capacity of STC 600 (Figure 9).

\section{Friction coefficient level}

The coefficient of friction is decisive for the maximum achievable friction performance. The increased friction coefficient leads to higher friction performance, which means 


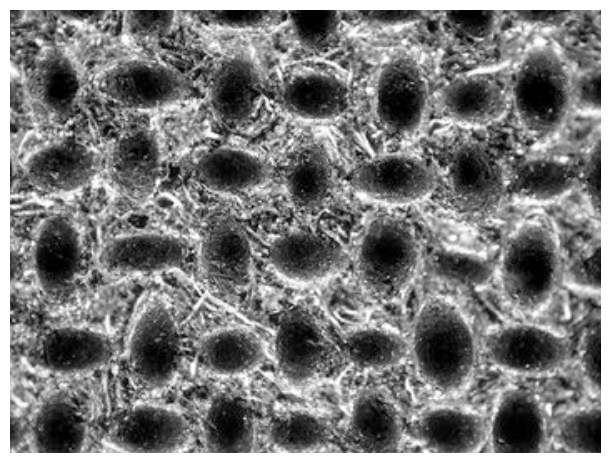

Figure 9 Surface structure of STC 600 friction lining

that the synchronization can occur within a shorter time. Values of 0.11 and above indicate that the STC 600 friction lining is a high-end product (Figure 10). Thus the friction material contributes to an increase in power density. Thanks to its high load capacity - both in absolute terms and relative to the benchmark - it is possible to reduce the necessary contact area and thus shorten the entire synchronization system. The gain in design envelope is added across all gear combinations and leads to a more compact and simpler transmission design.

Another indicator for quality is friction coefficient stability. This has practical rele-

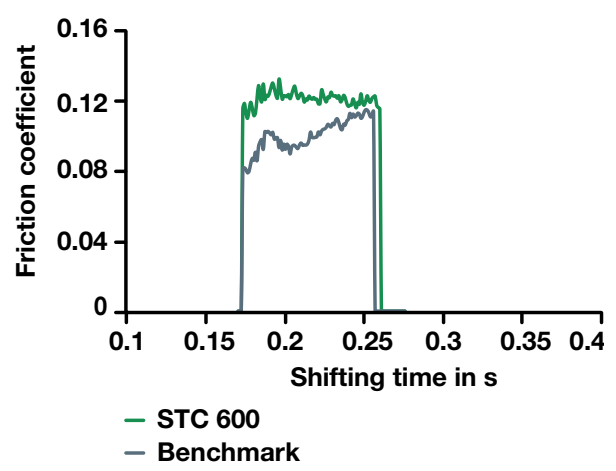

Figure 10 Convincing coefficient of friction in comparison with the benchmark

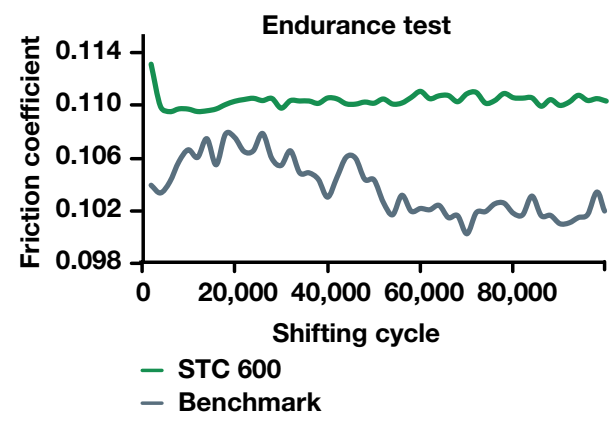

Figure 11 Low spread of coefficient of friction during the operating life

vance in that the gearshift feel remains the same over time. With STC 600, the start level remains practically unchanged over the entire operating life (Figure 11). The curve can be interpreted as a successful development outcome because a high friction coefficient and high friction stability are achieved at the same time. Technologically comparable materials statistically show a friction coefficient level of low uniformity over their operating life. In comparison with the materials commonly used on the market it is clear that the STC 600 friction lining is superior to those in particular in terms of friction coefficient gradients and curves (Figure 12).
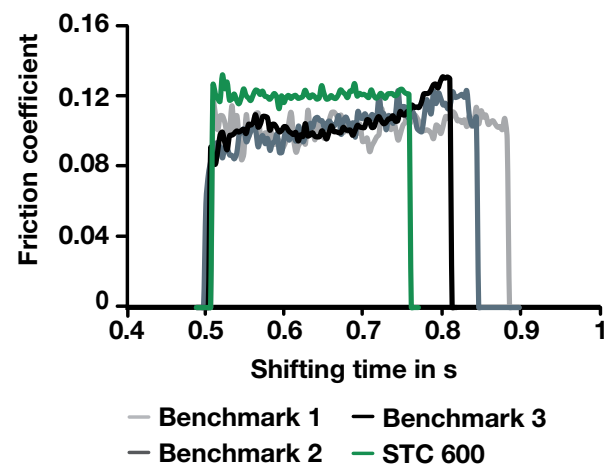

Figure 12 Friction coefficient gradient of various materials in comparison 


\section{Oil sensitivity and wear}

Depending on the oil, a friction material shows different behavior, in particular with respect to friction coefficient level and wear. A friction material is ideal from a customer's perspective, if it is equally efficient in all criteria in conjunction with any oil. In practice, this has not yet been achieved. When selecting the transmission oil, the primary focus is not usually on optimizing the gearshift comfort, but on protecting the gear teeth against wear and minimizing drag losses.

STC 600 friction lining shows relatively low sensitivity to the oils tested to date (Figure 13). The next development stage involves the extension of potential applications, for example, to a preferred type of transmission oil in a specific application. Schaeffler prefers to take this step hand in hand with the customer to ensure the best possible result.

When it comes to wear, STC 600 friction lining performs significantly better than the benchmark: Under the given experimental conditions and depending on the oil used, the necessary wear reserve for STC 600 needs to be only half as large, so that less installation space is required (Figure 14).

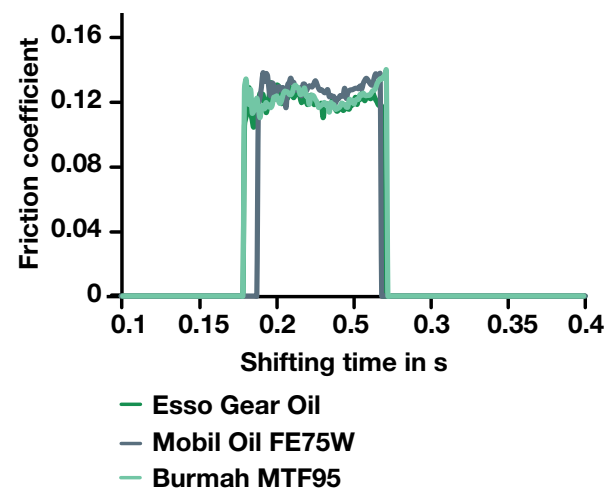

Figure 13 Low oil sensitivity of STC 600

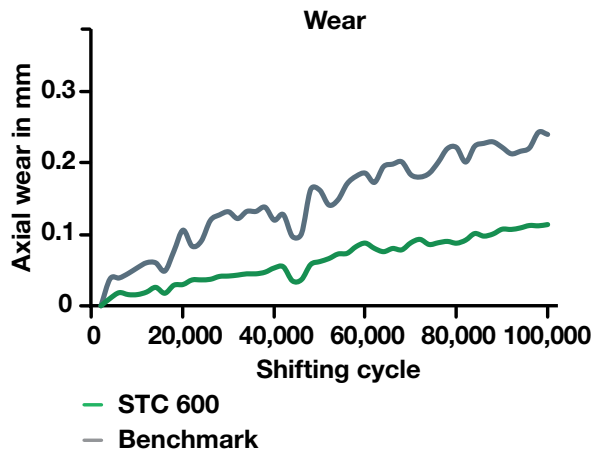

Figure 14 Low wear over the operating life

\section{Results in overview}

STC 300 and STC 600 have been designed for two different product categories. Both were developed by Schaeffler, starting with the selection of raw materials through to the finished product including the manufacturing processes, and they are manufactured using the company's own machines exclusively. The linings are positioned in different performance classes, but they all share the same carbon-based friction material. STC 300 offers higher performance in relation to friction coefficient stability and wear performance compared to brass and bronze-sintered products, but it comes at similar cost.

STC 600 achieves better results with regard to the essential criteria of dynamic friction coefficient, friction coefficient level and stability than the best products currently available on the market. In the combined analysis of friction coefficient stability over the operating life and the margin with which the different friction coefficients deviate from each other during the individual gearshifts, STC 600 is close to the optimum (Figure 15). Its sensitivity to the transmission oils tested so far is low. STC 600 also compares 


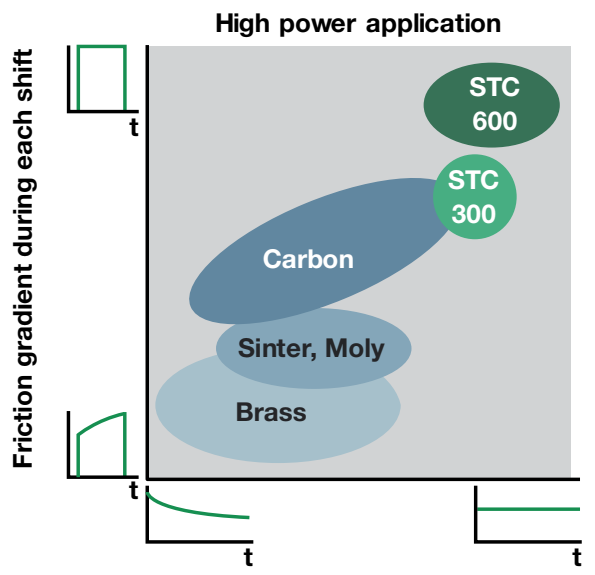

Friction level during life-cycle time

Figure 15 Overview of performance characteristics of STC 300 and STC 600

favorably to benchmark in terms of wear resistance.

\section{Innovative components with system impact}

\section{Smaller design envelope and lower weight}

The goal of designing systems and components that are as light as possible while maintaining the cost targets also applies to the transmission and its subsystems. In addition, the minimization of the required design envelope is gaining more and more importance. The "strut-in-sleeve" concept is a big step forward in this regard. The name refers to the consistent further development of the selector sleeve, detent and selector hub and the optimized harmonization of these components. If all options are used, then the mass of each synchronizer can be reduced by about $90 \mathrm{~g}$. With a six-speed transmission this comes to about $350 \mathrm{~g}$ - secondary effects at transmission level not included.

\section{Operating principle}

In the conventional design, the rib of the selector hub houses the pressure springs of the presynchronization detent. This installation space requires a specific mechanical strength which is achieved by appropriate material thickness. The further development of this basic design is a version using flat struts. These reduce the required depth of recess in the rib of the selector hub, which also reduces the stress peaks in the critical cross-section. In this way, higher torques can be transmitted with unchanged geometry. And vice versa: For an equally high transmission torque a narrower rib will suffice.

In principle, this approach would allow a narrower design for the entire selector hub and thus for the selector sleeve too. But since the shift path is a given, this op-

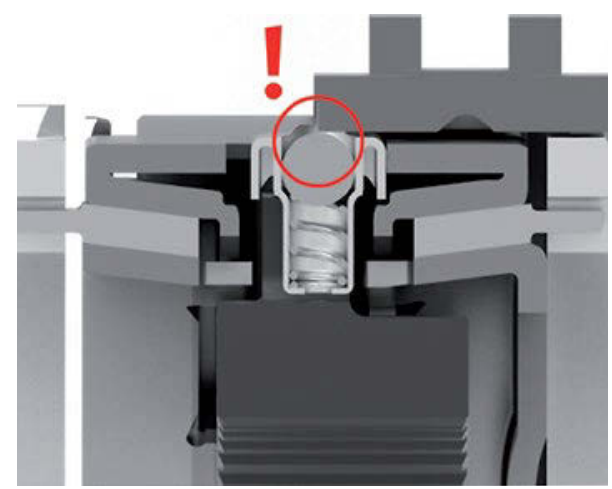

Figure 16 With conventionally assembled detents, the scope for producing a narrower selector hub design is limited 
tion is limited. This is because when the gear is engaged, there is a risk of the detent balls getting stuck since the selector sleeve no longer covers them completely (Figure 16).

The strut-in-sleeve concept path paves the way towards a narrower selector hub. In this case, the strut is not mounted in or on the selector hub, but in a recess in the internal teeth of the selector sleeve. So during the gearshift operation, it is now guided by the selector sleeve (Figure 17).

With this innovation, the selector hub is no longer impaired in any way so that there are no longer any critical crosssections. Now the width of the selector sleeve can be chosen freely and the opportunity of choosing a narrower rib can be fully exploited. This results in a farreaching benefit: Every single synchronization system is about $2 \mathrm{~mm}$ shorter. Consequently the gears move closer to each other. This, in turn, allows the use of shorter shafts and ultimately a shorter transmission housing.

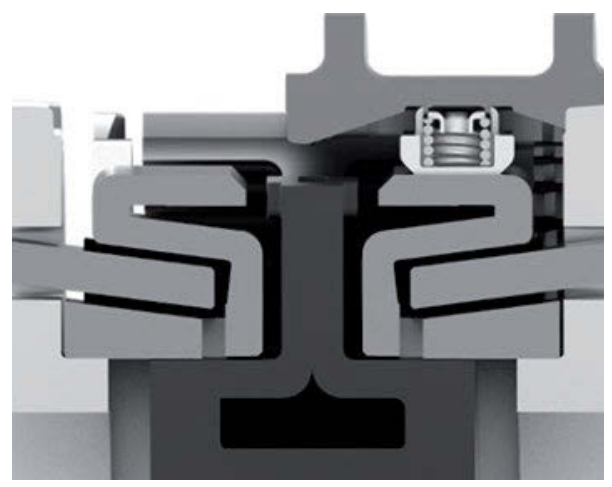

Figure 17 With a strut-in-sleeve selector sleeve, the strut is guided in the direction of the gearshift

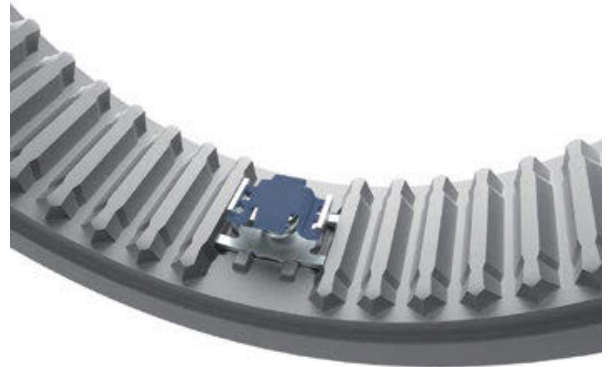

Figure 18 Selector sleeve with integrated detent (strut-in-sleeve)

\section{System requirements}

The strut-in-sleeve concept can be implemented at no additional cost. The basis for this is the selector sleeve manufactured by Schaeffler in volume production using forming methods. In contrast to components that are manufactured in a metal-cutting process from forged blanks, the integration of the usual three recesses for locating the struts does not require an additional operation. The recesses are designed so that the struts only have to be pushed in (Figure 18).

Selector hubs for car transmissions are now manufactured almost exclusively fromsintered metal. Since the selector sleeve introduces the torque into the selector hub off-center, it is subject to high torsional and bending loads. Therefore the selector hub has a solid design and weighs several hundred grams. New product concepts based on sheet steel designs focus on two courses of development: One on weight optimization and the other on strength optimization.

At the current state of development, the strength of the weight-optimized design (Figure 17) is still in the same range as that of a powder metal sintered component. In the case of a six-speed manual transmission in the $350 \mathrm{Nm}$ torque class, the weight advantage is about $350 \mathrm{~g}$, which is equivalent to $25 \%$ with regard to the synchronization units. 

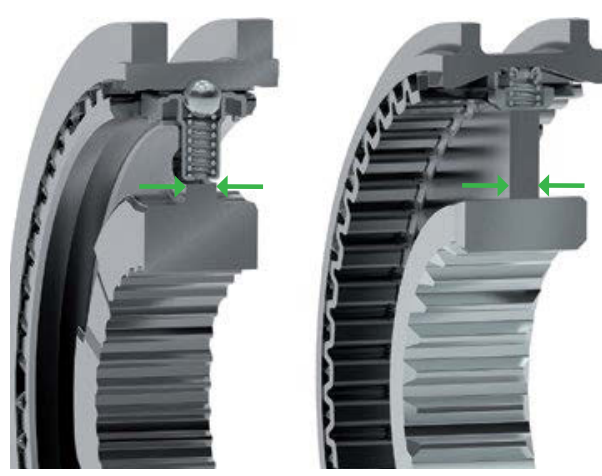

Figure 19 Selector hub of steel (right) compared to one made of sintered metal (left)

The strength potential of sheet steel designs can be used to reduce the design envelope. As a result, the rib width as well as the width of the internal teeth of the sheet steel selector hub can be reduced so that the slightly higher density of steel is over-compensated (Figure 19).

\section{Transmission savings}

Schaeffler has evaluated the possible secondary effects of a transmission optimized with strut-in-sleeve and improved synchro ring packages (Figure 20). For front transverse installations, the transmission housing is about $8 \mathrm{~mm}$ shorter due to shorter shafts. Depending on the conditions in the vehicle, this gain in design envelope can make a difference in compensating for the necessary enlargement of other components in the engine compartment. The weight savings from secondary effects alone add up to approximately $450 \mathrm{~g}$. Primary and secondary effects reduce the weight by about $800 \mathrm{~g}$.

In a longitudinally mounted transmission, the overall length is reduced by about $12 \mathrm{~mm}$. The secondary effect in terms of weight and material cost is roughly equivalent to that of a front transverse transmission.

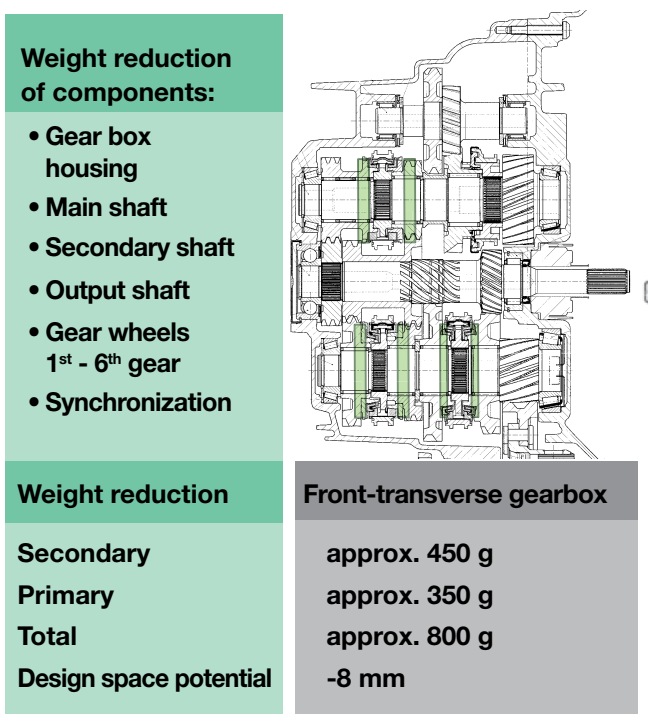

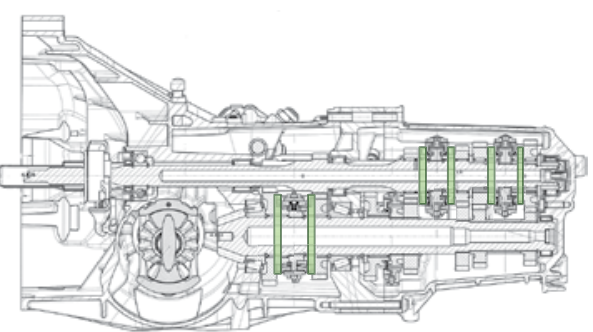

In-line gearbox

approx. $450 \mathrm{~g}$

approx. $350 \mathrm{~g}$

approx. $800 \mathrm{~g}$

$-12 \mathrm{~mm}$

Figure 20 Saving potential regarding weight and design envelope 


\section{Summary and outlook}

Customers in growth markets and increasingly in industrialized countries are looking for suppliers who offer not only individual components but rather complete synchronization systems for their transmissions. Schaeffler has thus decided to become a system supplier and has complemented its product portfolio with the development of carbon-based friction linings. It now consists of a sheet steel selector hub, selector sleeve, presynchronization detent, ring package and gear cone body. Schaeffler has developed all these components and manufacturing methods and manufactures them in-house worldwide.

In the course of this product range extension, the company has continuously developed the expertise necessary to design synchronization systems using an integrated approach. This involves not only the validation of the specific component characteristics, but also the functional optimization of power transmissions from the clutch to the speed gear including vibration isolation, in vehicle tests if necessary. Schaeffler has already demonstrated its expertise in this area in related projects.

In system optimization Schaeffler can draw on its long-standing expertise in component development. Thanks to the high degree of vertical integration, the components can be precisely matched to each other so that an optimum result is achieved at the system level. Concepts such as strutin-sleeve combined with a selector hub

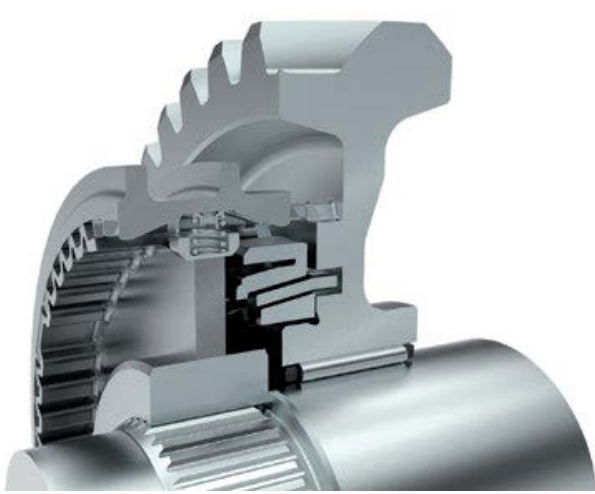

Figure 21 Further potential for reducing the design envelope can be tapped by including the freewheel and bearing support in the optimization of the synchronization system.

made from sheet steel illustrate the potential: The secondary effects of a shorter synchronization system result in a more compact and lightweight transmission.

The aim of a smaller design envelope will be given even more attention in the future. A development approach that extends beyond system boundaries opens up the opportunity of achieving even better results than the ones described above - for example, if the speed gear is included in the optimization of the synchronization system (Figure 21). However, designs that extend far beyond those currently encountered on the market require new solutions for the bearings and gears. Therefore, the company's combined expertise in the area of tooth systems and bearings is gaining increasing importance in the further development of synchronization systems. 